\title{
Superprism Phenomena in Planar Photonic Crystals
}

\author{
Lijun Wu, Michael Mazilu, Tim Karle, and Thomas F. Krauss
}

\begin{abstract}
We utilize the anomalous dispersion of planar photonic crystals near the dielectric band edge to control the wavelength-dependent propagation of light. We typically observe an angular swing of up to $10^{\circ}$ as the input wavelength is changed from $1290 \mathrm{~nm}$ to $1310 \mathrm{~nm}$, which signifies an angular dispersion of 0.5 degree/nm. Such a strong angular dispersion is of the order required for wavelength-division multiplexing systems. This is the first demonstration of the "superprism" effect in a planar configuration with a small lattice.
\end{abstract}

Index Terms-Angular dispersion, planar photonic crystals, superprism phenomena.

W AVELENGTH-DIVISION multiplexing (WDM) has become the established method of increasing the capacity and flexibility of the optical communications network. Typical multiplexers used today are based on arrayed waveguide gratings (AWGs), which occupy an area of several $\mathrm{cm}^{2}$ and have a capacity of 40 channels. Future networks will require hundreds of channels and therefore need much more compact devices, particularly if the multiplexers are to be combined with additional functionality, such as switching and driver electronics. Photonic crystals (PhCs), because of their interesting dispersive properties, especially near the photonic bandgap [1], [2], are promising candidates for WDM applications. Recently, Kosaka et al. demonstrated a highly dispersive photonic microstructure in a 3-D ("autocloned") $\mathrm{PhC}$, which was termed optical "superprism" [3]-[5]. The "autocloned" structure has very favorable properties, but requires specialized nonstandard fabrication techniques. Furthermore, the nature of the superprism effect does not require 3-D periodicity, and similar effects can be observed in 1-D and 2-D systems [6]. Moreover, the autocloned approach does not describe guided waves but rather beam propagation in a bulk-inhomogeneous-material. This will limit the usefulness of their structure for fabricating compact systems [7].

Planar PhCs, on the other hand, are much more suited for novel integrated optics applications because of their relative ease of fabrication compared to 3-D systems [8]. They use 2-D $\mathrm{PhCs}$ to confine and manipulate the light in-plane and total internal reflection to confine light vertically. Surprisingly, the superprism effect has so far, to our knowledge, not been studied in a planar 2-D system.

Previous work by Lin et al. demonstrated a prism-effect via refractive index modification of up to $20 \%$ within a frequency change of $30 \%$ (at radio frequency) for a 2-D photonic lattice

Manuscript received September 18, 2001; revised April 2, 2002. The work of T. F. Krauss was supported by a Royal Society University Research Fellowship. The authors are with The Ultrafast Photonics Collaboration, School of Physics and Astronomy, University of St. Andrews, St. Andrews, Fife KY16 9SS U.K. (e-mail: lw21@st-and.ac.uk).

Publisher Item Identifier S 0018-9197(02)05714-7.
[9]. However, this angular dispersion is only comparative to that of a conventional grating. The significant difference between Kosaka's and Lin's results is in the physical understanding of the angular dispersion phenomenon. Kosaka et al. recognized the anisotropy in the dispersion relationship and determined the light propagation direction via the group velocity, using wavevector diagrams discussed in detail by Russel [10]. In contrast, Lin et al. assumed isotropic dispersion and ascribed the angular dispersion to the modification of the refractive index. Here, by studying the iso-frequency contours of a semiconductor waveguide $\mathrm{PhC}$ in the similar way as that of Kosaka, we succeeded in demonstrating the superprism effect in a planar configuration. The angular dispersion is almost an order of magnitude larger than that estimated by Lin and two orders of magnitude larger than that of conventional prisms. This result reaches a tenth of Kosaka's angular dispersion, obtained in their 3-D "autocloned" system. The main difference between Kosaka's and our results lies at the operating point. Kosaka et al. operated their superprism near the $\Gamma$ point, which means the $\mathrm{k}$ vector has a large component perpendicular to the plane of the crystal. This is possible because of the 3-D confinement offered by the autocloned system. In contrast, we have to operate near the symmetry point ( $K$ or $M$ in the hexagonal case), because the vertical confinement offered by our waveguide is relatively weak, so $\mathrm{k}$ has to have its main component in the plane of the crystal.

Our system consists of a GaAs-based heterostructure perforated by a triangular $\mathrm{PhC}$ lattice. The sample was fabricated by electron-beam lithography and reactive-ion etching [8]. The lattice constant of the hole array $a$ is designed as $280 \mathrm{~nm}$ and the hole diameter $d$ is $190 \mathrm{~nm}$. This ratio between the hole size and the lattice was shown to be successful in previous experiments [8]. The 240-nm-thick GaAs slab has a 200-nm-thick $\mathrm{SiO}_{2}$ mask on the top and a 2000 -nm-thick $\mathrm{Ga}_{0.1} \mathrm{Al}_{0.9}$ As layer as the lower cladding. The input waveguide, which was chosen to be $10 \mu \mathrm{m}$ wide to avoid strong diffraction at its output and to generate a quasi-parallel input for the superprism, is tilted at $25^{\circ}$ from normal to the crystal edge (the incident edge is $\Gamma-M$ ). The shape for the crystal area was designed as a semicircle to ensure the light to be transported out straightforward from the crystals. The $10-\mu \mathrm{m}$ output waveguides designed to pick up the output light are oriented at divisions of $10^{\circ}$ from $-70^{\circ}$ to $70^{\circ}$, which leads to the large space between the crystal area and the output waveguide. Fig. 1(a) and (b) show the scanning electron microscope (SEM) pictures of the top views for PhCs with input and output waveguides and the interface between the input waveguide and PhCs.

The schematic diagram for our measurement setup is shown in Fig. 2. The semiconductor laser (Agilent 8167B) has a tunable wavelength range extending from 1255 to $1365 \mathrm{~nm}$. The light is injected in TE-polarization ( $H$-field parallel to etched holes) 

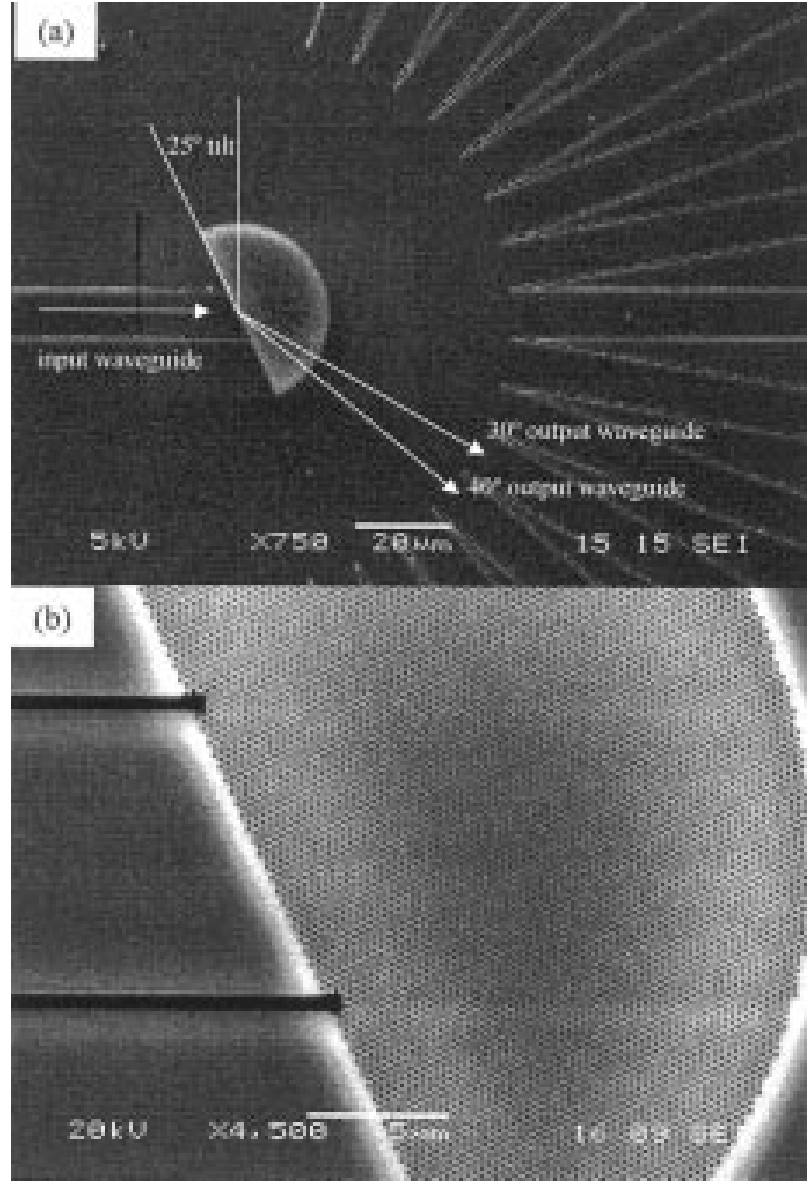

Fig. 1. (a) SEM top view of $\mathrm{PhCs}$ with input and output waveguides. The input waveguide is tilted $25^{\circ}$ from normal to the crystal edge (the incident edge is $\Gamma-M)$. The output waveguides are orientated at divisions of $10^{\circ}$ from $-70^{\circ}$ to $70^{\circ}$. (b) The interface between the input waveguide and $\mathrm{PhCs}$

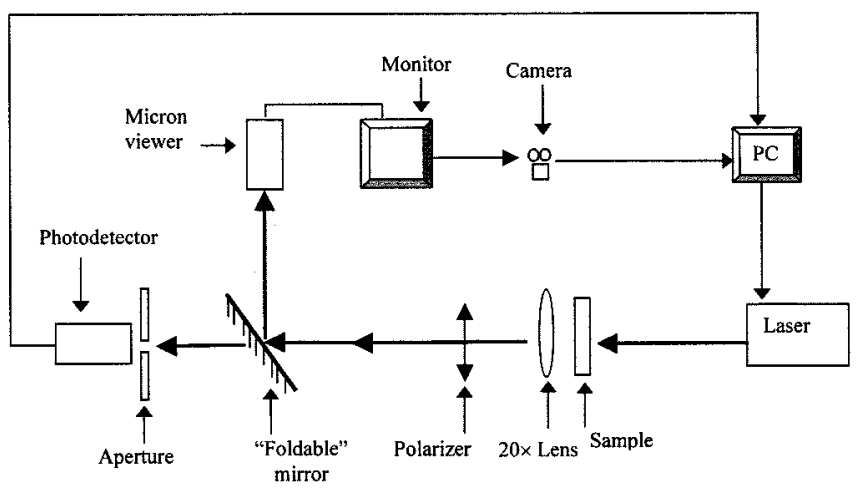

Fig. 2. Schematic diagram of the measuring setup.

via a cleaved single-mode fiber. A Vidicon camera is used to observe the output facet of the sample. In Fig. 3, video images of the output facet of the sample are shown at different input wavelengths. The observed images indicate that the light swings from the $30^{\circ}$ to the $40^{\circ}$ waveguide as the input wavelength is changed from $1290 \mathrm{~nm}$ to $1310 \mathrm{~nm}$ [Fig. 3(a)]. The multiple spots in each waveguide here are due to higher order modes excited in the $10 \mu \mathrm{m}$ wide output waveguides. Note that when the wavelength is between $1295 \mathrm{~nm}$ and $1305 \mathrm{~nm}$, there are spots in both the $30^{\circ}$ and $40^{\circ}$ waveguides. The possible explanation is that the swing of the light in this range is less than $10^{\circ}$ and the direction of propagation is somewhere between the $30^{\circ}$ and the $40^{\circ}$ waveguide, so the signal couples into both waveguides. The transmission from the $\mathrm{PhCs}$ is approximately $1 \%$ if the output intensity is normalized to that of a blank waveguide.

The physical mechanism behind this effect can be explained with the iso-frequency contours of the photonic band structure. Fig. 4(a) shows the photonic band structure (TE-polarization) for hexagonal lattices calculated by the plane-wave expansion method. The units for the frequency $\nu$ are c/a. The iso-frequency dispersion surfaces at frequencies of 0.214 and 0.217 were then calculated from the band structure in various directions [Fig. 4(b)], using a refractive index of 3.10 for the semiconductor host. This index represents the effective index of the waveguide slab and was verified by identifying the photonic band edge in separate transmission measurements. The filling ratio is approximated as 30\%, from SEM micrographs. Given an incident wavevector, the propagation direction is obtained through the momentum conservation rule ${ }^{1}$ and the concept of the group velocity $\nu_{g}=\nabla_{\kappa} \omega(\kappa)$ being normal to the iso-frequency contours [10]. Note that, near the bandgap, the propagation direction will be changed acutely even if there is just a very small variation of the incident angle [Fig. 4(b)]. Furthermore, given a very small change for the input wavelength (frequency in the wavevector diagram), the shape of the iso-frequency curves experiences a large alteration. In Fig. 4(b), when the frequency is altered from 0.214 to 0.217 , which corresponds to a wavelength change from $1310 \mathrm{~nm}$ to $1290 \mathrm{~nm}$, the shape of the iso-frequency contour varies from a quasihexagram to a quasi-triangle.

In order to explain our results, we proceeded as follows. The incident light is oriented along the horizontal direction. The Brillouin zone of the crystal is rotated in order to reflect the angle of incidence and represents the geometry shown in Fig. 1. Using the construction line method described above, we should then be able to find the directions of the output light from the intersections with the iso-frequency contours. Unfortunately, this was not straightforward and did not yield success immediately. Even when accounting for a reasonable experimental error in all parameters involved, we could not explain the observed angular dispersion from the simple model. We then remeasured the waveguides and found that the results depended, in part, on the alignment between the input fiber and the $10-\mu \mathrm{m}$ semiconductor waveguide that feeds into the superprism. This is emphasized in Fig. 3(b), which shows a higher angular dispersion when the input fiber is tilted slightly. This gave us the critical clue: the input waveguide has a few imperfections on it, which may favor a few specific higher order modes. Higher order modes can be understood to propagate along a zigzag path and therefore do not enter the crystal at the normal angle. By scanning through the different possible angles, we then found that a propagation angle of the waveguided light of $23^{\circ}$ to the normal yields the observed swing from $30^{\circ}$ to $40^{\circ}$ in the superprism described

${ }^{1}$ Momentum conservation rule: the incident wavevector $k_{i}$ and propagation wavevector $k_{p}$ are continued for the tangential components parallel to the incident crystal edge, i.e., $k_{p / /}=k_{i / /}$. 


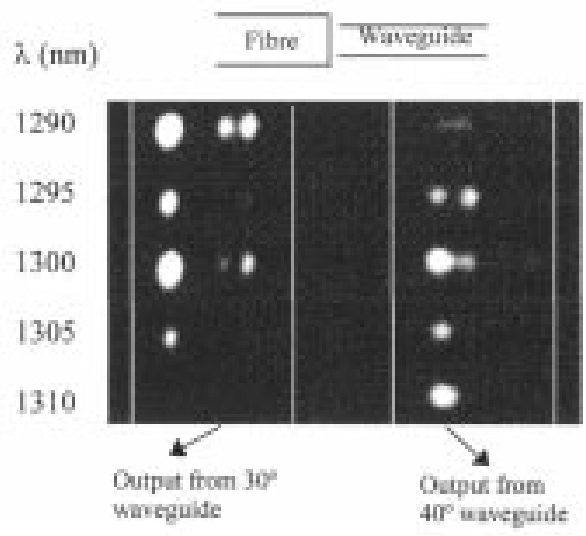

(a)
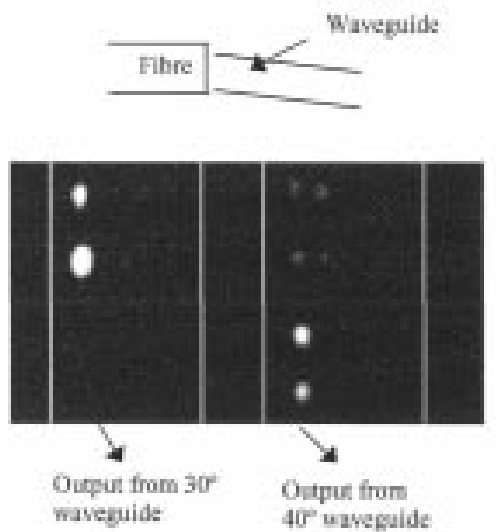

(b)

Fig. 3. Vidicon photographs for the output light spots at different wavelengths. The multiple spots in each waveguide are due to their multimoded-ness. (a) The input waveguide matches well with the fiber (see the top of the picture). We can see that the light swings from the $30^{\circ}$ to the $40^{\circ}$ waveguide as the input wavelength is changed from 1290 to $1310 \mathrm{~nm}$. (b) Sample is rotated by about 8 degrees with respect to the cleaved facet. The same swing is observed for a smaller change in wavelength, i.e., from 1300 to $1305 \mathrm{~nm}$. This can be explained with a steeper input angle than that shown in Fig. 4(b), and a projection onto the more dispersive part of the equi-frequency contour.

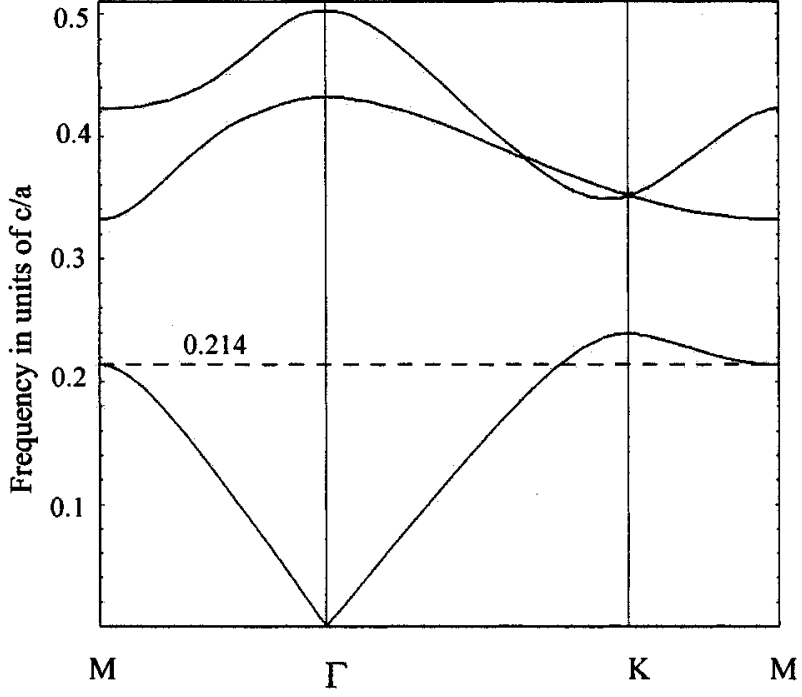

(a)

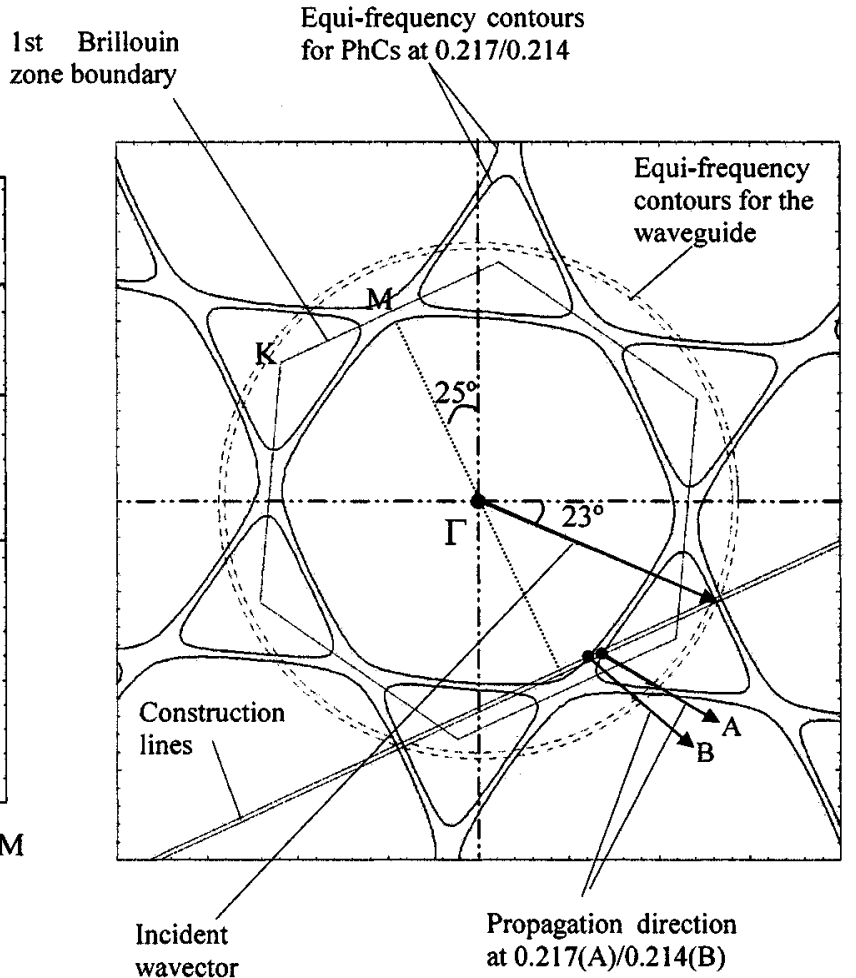

(b)

Fig. 4. (a) Photonic band structure (TE-polarized) for the PhCs used in this demonstration. A plane-wave expansion method was used. The dashed horizontal line corresponds to one of the frequencies we used in the experiment. (b) The iso-frequency contours for the first band (TE-polarized). The method used to derive the propagation direction is as follows: given the incident angle, the propagation wavevector is obtained by the momentum conservation rule. The propagation direction is then determined as being normal to the dispersion surface at the end point of the propagation wavevector. The solid lines are the equi-frequency contours for the PhCs and the dashed lines represent the uniform semiconductor waveguides. The iso-frequency contours were rotated by $25^{\circ}$ to match with the experiment situation, where the incident edge $\Gamma-M$ is tilted at $25^{\circ}$ from the vertical direction (Fig. 1). From this picture, it can be seen that, when the wavelength is altered by $20 \mathrm{~nm}$ (the frequency changes from 0.217 to 0.214 ), the propagation direction swings by $10^{\circ}$ (from $\mathrm{A}$ to $\mathrm{B}$ ) assuming the input mode is incident at $-23^{\circ}$ to the direction of the waveguide.

above. Considering the size $(10 \mu \mathrm{m})$ and refractive index contrast $(>3: 1)$ of the waveguide, a zigzag path of $23^{\circ}$ does not appear unreasonable and is well within the range allowed by total internal reflection. By adjusting the model, and assuming that the incident light was at $23^{\circ}$ to the waveguide normal, we were able to explain our observation after all. In Fig. 4(b), assuming the input mode is incident at $23^{\circ}$, it impinges on the superprism as shown and yields output angles of $30^{\circ}$ and $40^{\circ}$ for the frequencies corresponding to the wavelengths 1290 and $1310 \mathrm{~nm}$.

We wish to emphasize that the above explanation contains an assumption concerning waveguide mode propagation that we cannot prove conclusively. Assuming zigzag-like propagation 
of the mode in the ridge waveguide that feeds into the superprism is the only explanation, however, that allows us to project the input wavevector onto the high-dispersion part of the iso-frequency contour, thus explaining our experimental result. Furthermore, this assumption does not detract from the exciting observation of high angular dispersion in a planar superprism. Also, it highlights the fact that the properties of the access waveguide must be taken into account.

Finally, the $10^{\circ}$ light swing for the change of wavelength from $1290 \mathrm{~nm}$ to $1310 \mathrm{~nm}$ corresponds to a $0.3^{\circ}$ light swing when the wavelength is changed from $1550 \mathrm{~nm}$ to $1550.8 \mathrm{~nm}$ (ITU grid for 100-GHz separation). To project the possible performance of the superprism in a WDM system, we extrapolated our result as follows. For a separation of the output waveguides of $3 \mu \mathrm{m}$, the device would have to be $500 \mu \mathrm{m}$ long. Assuming the beam was collimated, it could be coupled into $\mathrm{PhC}$ waveguides separated by $3 \mu \mathrm{m}$ and such waveguides would have a typical crosstalk of approximately $30 \mathrm{~dB}$. Therefore, the angular dispersion of 0.5 -degree/nm observed in our superprism is of the order required for WDM systems. Furthermore, being realized in a 2-D structure, this superprism is easily combined with other planar optical components such as ridge waveguides, $60^{\circ}$ bends and $\mathrm{Y}$ couplers, which makes it attractive for applications in ultracompact photonic integrated circuits. Currently, we are improving the design of the input and output waveguides to avoid the observed multimoded character and optimizing superprism parameters to increase the wavelength range over which strong dispersion is observed.

To summarize, the anomalous dispersion near the $\mathrm{PhC}$ band edge was utilized to control the wavelength-dependent propagation in a planar 2-D photonic lattice. Strong angular dispersion of up to 0.5 -degree/nm was obtained, but only over a relatively small wavelength range and with compromised beam quality, both of which we are currently addressing. Furthermore, we highlighted the fact that taking the properties of the access waveguide into account is critical, i.e., one cannot simply assume equivalence between the plane wave and waveguide modes. Overall, we consider this first demonstration of the superprism effect in a planar configuration as a major step toward the realization of ultracompact photonic integrated circuits.

\section{ACKNOWLEDGMENT}

The authors gratefully acknowledge the technical support of the Nanoelectronics Research Centre and I. Ntakis at Glasgow University. The authors would also like to thank Dr. R. Wilson, D. Brown, and Dr. A. Tahraoui for useful discussions and G. Robb for invaluable help with the experiments. They also wish to thank Dr. P. Stavrinou of Imperial College, London, U.K., for the provision of the initial plane wave code.

\section{REFERENCES}

[1] M. Notomi, "Theory of light propagation in strongly modulated photonic crystals: Refractionlike behavior in the vicinity of the photonic band gap," Phys. Rev. B, vol. 62, no. 16, pp. 10 696-10705, 2000.

[2] B. Gralak, S. Enoch, and G. Tayeb, "Anomalous refractive properties of photonic crystals," J. Opt. Soc. Amer. A, vol. 17, no. 6, pp. 1012-1020, 2000 .
[3] H. Kosaka, T. Kawashima, A. Tomita, M. Notomi, T. Tamamura, T. Sato, and S. Kawakami, "Superprism phenomena in photonic crystals: Toward microscale lightwave circuits," J. Lightwave Technol., vol. 17, pp. 2032-2038, Nov. 1999.

[4] - "Photonic crystals for micro lightwave circuits using wavelength-dependent angular beam steering," Appl. Phys. Lett., vol. 7, pp. 1370-1372, 1999.

[5] - "Superprism phenomena in photonic crystals," Phys. Rev. B, vol. 58, no. 16, pp. R10096-R10099, 1998.

[6] R. Zengerle, "Light propagation in singly and doubly periodic planar waveguides," J. Mod. Opt., vol. 34, no. 12, pp. 1589-1617, 1987.

[7] E. Silvestre, J. M. Pottage, P. St. J. Russel, and P. J. Roberts, "Design of thin-film photonic crystal waveguides," Appl. Phys. Lett., vol. 77, no. 7, pp. 942-944, 2000.

[8] T. F. Krauss, R. M. De La Rue, and S. Brand, "Two-dimensional photonic-bandgap structures operating at near-infrared wavelengths," $\mathrm{Na}$ ture, vol. 383, no. 24, pp. 699-702, 1996.

[9] S. Y. Lin, V. M. Hietala, L. Wang, and E. D. Jones, "Highly dispersion photonic band-gap prism," Opt. Lett., vol. 21, no. 21, pp. 1771-1773, 1996.

[10] P. St. J. Russell, T. A. Birks, and F. D. Lloyd-Lucas, "Photonic Bloch waves and photonic band gaps," in Confined Electrons and Phonons. ser. NATO Advanced Studies Institute Series B: Physics, E. Burstein and C. Weisbuch, Eds. New York: Plenum, 1995, vol. 340, pp. 585-633.

Lijun Wu received the B.S. degree from Hunan University, China, in 1994, the M.S. degree from Beijing Polytechnic University, Beijing, China, in 1997, and the Ph.D. degree from the Institute of Physics, Chinese Academy of Sciences, in 2000 .

In October of 2000, she joined the University of St. Andrews, St. Andrews, U.K., as a Research Fellow and started research in 2-D photonic crystals. Her current interest is to use photonic crystals to control light from spatial and spectral dispersion and thereby act as "prisms" in multiwavelength systems.

Michael Mazilu was born in Bucharest, Romania, in 1969. He received the degree in condensed matter and material physics from the University of Louis Pasteur, Strasbourg, France, in 1993, and the Ph.D. degree from the University of St. Andrews, St. Andrews, U.K., in 2000.

His research activities are focused on modeling and characterization of light propagation through linear and nonlinear devices.

Tim Karle received the M.Phys. (Hons) degree in physics from the Universityof Manchester, Manchester, U.K., in 1999 and the M.Sc. degree in optoelectronic and laser devices with distinction jointly from Heriot Watt University, Edinburgh, U.K., and St. Andrews University, St. Andrews, U.K., in 2000. He is currently working toward the Ph.D. degree at the University of St. Andrews.

He was a Design Engineer with Renishaw Plc., Rnishaw, Gloucestershire, U.K., conditioning diode laser light for Raman spectroscopy. He joined the Photonic Band Gap Research Group at St. Andrews in 2001, and is currently researching the dispersive properties of 2-D photonic bandgap structures for integrated optics.

Mr. Karle was the recipient of the Neil Forbes/Scottish Enterprise Prize for excellence in Optoelectronics during his graduate studies.

Thomas F. Krauss received the Ph.D. degree from the University of Glasgow, Glasgow, U.K., where his work focused on semiconductor ring lasers.

He has been involved in integrated optics research since 1989. While at the University of Glasgow, he demonstrated low-threshold current levels and greatly improved external device efficiency. In 1993, he started work on photonic crystals via a series of fellowships (EPSRC and Royal Society in 1993 and 1995-2003, respectively) and became one of the pioneers of semiconductor-based photonic crystals. In 1997, he spent one year at the California Institute of Technology, Pasadena, working on efficient photonic crystal based light emitters. In 2000, he moved to a personal chair in Optoelectronics at the School of Physics and Astronomy, University of St. Andrews, and set up a photonic crystal research group (currently ten members) and a semiconductor microfabrication facility. He is grant holder of several U.K. research projects and is coordinator of the European research project "PICCO." He was organizer and chairman of the trend-setting Workshop "PECS3" in St. Andrews, June 2001 and is on the committee of several other workshops and summer schools, including MRS and PECS.

Dr. Krauss is a Fellow of the Royal Society of Edinburgh. 